\title{
Gold(I)-Catalyzed Enantioselective Intramolecular Hydroamination of Allenes with Ureas
}

\author{
Hao Li, Seong Du Lee, and Ross A. Widenhoefer* \\ French Family Science Center, Duke University, Durham, North Carolina, USA
}

\begin{abstract}
Enantioselective intramolecular hydroamination of $N$-allenyl ureas catalyzed by an enantiomerically enriched bis(gold) phosphine complex forms pyrrolidine derivatives in good yield with up to $93 \%$ ee.
\end{abstract}

\section{Keywords \\ gold; hydroamination; catalysis; allene; pyrrolidine}

\section{Introduction}

The transition-metal-catalyzed addition of the $\mathrm{N}-\mathrm{H}$ bond of an amine or amide derivative across the $\mathrm{C}=\mathrm{C}$ bond of an allene (hydroamination) represents an efficient and atom economical approach to the synthesis of cyclic and acyclic amines [1]. Although efficient methods for both the intermolecular [2] and intramolecular [3] hydroamination of allenes have been developed, only recently have effective methods for the enantioselective hydroamination of allenes appeared that employ chiral bis(gold) phosphine complexes as catalysts [4-10]. For example, we have reported the gold(I)-catalyzed enantioselective, intramolecular hydroamination of allenes with carbamates to form vinyl pyrrolidines with up to $96 \%$ ee [6,7]. Similarly, Toste and co-workers have reported the enantioselective intramolecular hydroamination of allenes with sulfonamides [8] and, more recently, hydrazines and hydroxylamines [9] with up to $99 \%$ ee.

We have found ureas to be particularly effective nucleophiles for the gold(I)-catalyzed intra[11] and intermolecular [12] hydroamination of alkenes and for the intramolecular dihydroamination of $N$ - $\delta$-allenyl ureas to form bicyclic imidazolidin-2-ones [13]. With the objective of expanding the nucleophile scope of gold(I)-catalyzed enantioselective allene hydroamination, we considered the application of ureas as nucleophiles in these transformations. Here we provide an account of our efforts directed toward the development of a gold (I)-catalyzed procedure for the enantioselective intramolecular hydroamination of allenes with ureas.

\section{Results and discussion}

We began our efforts in the area of catalytic hydroamination of allenes with ureas employing a catalyst system similar to that employed for the intramolecular hydroamination of allenes with carbamates [6]. In an initial experiment, treatment of $N$ - $\gamma$-allenyl urea (1) with a catalytic 1:2 mixture of $[(S)-2] \mathrm{Au}_{2} \mathrm{Cl}_{2}[\mathbf{2}=\mathrm{DTBM}-\mathrm{MeObiphep}]$ and $\mathrm{AgBF}_{4}$ in

\footnotetext{
"Corresponding author. Tel: (919) 660-1533; fax: (919) 660-1605; ross.widenhoefer@duke.edu.
} 
dioxane at room temperature for $36 \mathrm{~h}$ gave the 2-vinylpyrrolidine $\mathbf{3}$ with good enantioselectivity ( $88 \%$ ee) but with only $\sim 30 \%$ conversion (Table 1 , entry 1 ). In an effort to enhance the activity of the gold(I) catalyzed hydroamination, we evaluated the hydroamination as a function of solvent. While employment of toluene, THF, methanol, $\mathrm{CH}_{2} \mathrm{Cl}_{2}$, chloroform, or acetonitrile led to poor conversion and/or poor enantioselectivity (Table 1, entries 2-7), use of diethyl ether as solvent led to good conversion $(80 \%)$ with excellent enantioselectity (93\% ee) (Table 1 , entry 8). Interestingly, neither $N$ - $\gamma$-allenyl urea $\mathbf{1}$ nor the vinyl pyrrolidine $\mathbf{3}$ was fully soluble in diethyl ether, which suggests that low substrate concentration facilitates the high enantioselectivity of hydroamination.

In a preparative-scale experiment, treatment of 1 with a catalytic 1:2 mixture of $[(S)-2] \mathrm{Au}_{2} \mathrm{Cl}_{2} / \mathrm{AgBF}_{4}$ in diethyl ether at room temperature for $48 \mathrm{~h}$ led to isolation of 2vinylpyrrolidine 1 in $90 \%$ yield with $93 \%$ ee (Table 2, entry 1). Employment of $\mathrm{AgBF}_{4}$ cocatalyst was required to generate the catalytically active dicationic bis gold(I) complex, but silver alone was not an active catalyst for the conversion of $\mathbf{1}$ to $\mathbf{3}$. For example, treatment of $1(0.10 \mathrm{M})$ with either $[(S)-2] \mathrm{Au}_{2} \mathrm{Cl}_{2}(5 \mathrm{~mol} \%), \mathrm{AgBF}_{4}(10 \mathrm{~mol} \%)$, or a mixture of $(S)$-2 (5 $\mathrm{mol} \%)$ and $\mathrm{AgBF}_{4}(10 \mathrm{~mol} \%)$ in diethyl ether at room temperature for $48 \mathrm{~h}$ led, in each case, to no consumption of $\mathbf{1}$ and no detectable formation of 3. $N$ - $\gamma$-allenyl ureas that possessed either an electron-rich or electron-deficient $N^{\prime}$-aryl substituent or an $N^{\prime}$-alkyl group underwent gold(I)-catalyzed enantioselective hydroamination in excellent yield but with diminished enantioselectivity relative to 1 (Table 2, entries 2-7). Similarly, an $N-\gamma$ allenyl urea that possessed a terminally dimethyl-substituted allenyl moiety and an $N-\delta$ allenyl urea underwent gold(I)-catalyzed hydroamination in good yield with modest enantioselectivity (Table 2, entries 8 and 9 ). Although we have previously demonstrated the room temperature dihydroamination of $N$ - $\delta$-allenyl ureas catalyzed by achiral mono(gold) phosphine complexes [13], no bicyclization was observed employing the [(S)-2] $\mathrm{Au}_{2} \mathrm{Cl}_{2} /$ $\mathrm{AgBF}_{4}$ catalyst system.

Enantioselective hydroamination was highly sensitive to substitution along the alkyl chain that tethered the allenyl moiety to the urea nucleophile. Substitution of the gem-diphenyl groups of $\mathbf{1}$ with either a cyclohexyl group or hydrogen atoms led to a precipitous drop in the enantioselectivity of hydroamination (Table 2, entries 10 and 11). Although cyclization reactions are often facilitated by gem-disubstitution along the main chain (Thorpe-Ingold effect) [14], the pronounced effect of backbone substitution on the enantioselectivity of gold(I)-catalyzed hydroamination does not appear to be due to the rates of cyclization as both the cyclohexyl-substituted and unsubstituted $\gamma$-allenyl ureas cyclized with rates similar to that of $\mathbf{1}$. Rather, it appears that the gem-diphenyl substitution of $\mathbf{1}$ and related $\gamma$-allenyl ureas enhance the stereoselectivity of the binding of the chiral bis(gold) catalyst to the prochiral allene. In any event, similar behavior was observed in the gold(I) catalyzed enantioselective intramolecular hydroamination of allenes with carbamates [6,7] and in the intramolecular hydroalkoxylation of allenes with alcohols [15].

We have previously demonstrated the gold(I)-catalyzed dynamic kinetic enantioselective hydroamination (DKEH) of $N$ - $\gamma$-allenyl carbamates that possessed an axially chiral trisubstituted allenyl moiety [7] and we sought to determine if DKEH was applicable to $N$ - $\gamma$ allenyl ureas. Unfortunately, treatment of the racemic $N$-allenyl urea 4 that possessed an axially chiral allenyl moiety with a catalytic mixture of $[(S)-2] \mathrm{Au}_{2} \mathrm{Cl}_{2}$ and $\mathrm{AgBF}_{4}$ in diethyl ether led to no conversion after $16 \mathrm{~h}$ at room temperature, presumably due to the poor solubility of $\mathbf{4}$ in ether (Table 3, entry 1). In comparison, gold(I)-catalyzed hydroamination of $\mathbf{4}$ in the more solubilizing solvent chloroform at room temperature for $16 \mathrm{~h}$ led to complete conversion to form vinyl pyrrolidine $\mathbf{5}$ as an inseparable 3.0:1 mixture of diastereomers with $55 \%$ and $33 \%$ ee for the major and minor diastereomers, respectively (Table 2, entry 2) [16]. Interestingly, employment of mixtures of diethyl ether and 
chloroform as solvent proved superior to either solvent alone (Table 3, entries 3-6). A 3:1 mixture of ether and chloroform proved optimal, leading to complete consumption of $\mathbf{4}$ after $16 \mathrm{~h}$ at room temperature to form $\mathbf{5}$ as a 2.9:1 mixture of diastereomers with $88 \%$ and $85 \%$ ee for the major and minor diastereomers, respectively (Table 3, entry 4). In comparison, hydroamination of $\mathbf{4}$ in 3:1 ether:chloroform at $0{ }^{\circ} \mathrm{C}$ for $36 \mathrm{~h}$ led to complete consumption of $\mathbf{4}$ and isolation of $\mathbf{5}$ in $93 \%$ yield as a 1.3:1 mixture of diastereomers with $\geq 90 \%$ ee for both diastereomers (Table 3, entry 7). The near $\sim 1: 1$ mixture of diastereomers formed at $0{ }^{\circ} \mathrm{C}$ indicates that allene racemization is slow relative to cyclization under these conditions and that hydroamination occurs predominantly through a static catalyst-controlled pathway. Similar behavior was observed for the gold(I)-catalyzed enantioselective intramolecular hydroalkoxylation of chiral $\gamma$-allenyl alcohols [17].

\section{Conclusions}

In summary, we have developed a gold(I)-catalyzed protocol for the enantioselective hydroamination of achiral $\mathrm{N}$-allenyl ureas to form 2-vinyl pyrrolidines with up to $93 \%$ ee. Similarly, allenyl ureas that possessed an axially chiral allenyl moiety underwent hydroamination with high enantioselectivity/low diastereoselectivity in a catalyst controlled pathway. We continue to work toward expanding the scope of gold(I)-catalyzed allene hydroamination and toward the identification of more selective and more general catalysts for enantioselective hydroamination.

\section{Experimental}

\subsection{General methods}

Catalytic reactions were performed in sealed glass tubes under an atmosphere of dry nitrogen unless noted otherwise. NMR spectra were obtained on a Varian spectrometer operating at $400 \mathrm{MHz}$ for ${ }^{1} \mathrm{H} \mathrm{NMR}$ and $100 \mathrm{MHz}$ for ${ }^{13} \mathrm{C}$ NMR in $\mathrm{CDCl}_{3}$ unless noted otherwise. IR spectra were obtained on a Bomen MB-100 FT IR spectrometer. Gas chromatography was performed on a Hewlett-Packard 5890 gas chromatograph equipped with a $25 \mathrm{~m}$ polydimethylsiloxane capillary column. Chiral HPLC of pyrrolidine $\mathbf{3}$ was performed on a Hewlett-Packard chromatograph quipped with a $0.46 \mathrm{~cm} \times 15 \mathrm{~cm}$ Chiralcel OD-H column; chiral HPLC of all other $N$-heterocycles utilized a $0.46 \mathrm{~cm} \times 25 \mathrm{~cm}$ Chiralpak AD-H column. Flash column chromatography was performed employing 200-400 mesh silica gel (EM). Thin layer chromatography (TLC) was performed on silica gel 60 F254. Elemental analyses were performed by Complete Analysis Laboratories (Parsippany, $\mathrm{NJ}) . \quad N$ - $\gamma$-allenyl urea 1, 1-(2,2-diphenylhepta-5,6-dienyl)-3-(4-nitrophenyl)urea, and 1(hexa-4,5-dienyl)-3-(4-nitrophenyl)urea were synthesized employing a published procedure [13]. Remaining $N$-Allenyl ureas were synthesized employing an analogous procedure (see Supporting Information).

\section{2. $\mathrm{N}$-Heterocycles}

4.2.1. $\mathrm{N}$-(4-Nitrophenyl)-4,4-diphenyl-2-vinylpyrrolidine-1-carboxamide (3)-A suspension of $1(21 \mathrm{mg}, 0.05 \mathrm{mmol}),[(S)-2] \mathrm{Au}_{2} \mathrm{Cl}_{2}\left(4.0 \mathrm{mg}, 2.5 \times 10^{-3} \mathrm{mmol}\right)$, and $\mathrm{AgBF}_{4}$ $\left(1.0 \mathrm{mg}, 5.0 \times 10^{-3} \mathrm{mmol}\right)$ in diethyl ether $(0.5 \mathrm{~mL})$ was stirred at room temperature for 36 h. The crude reaction mixture was loaded directly onto a silica gel column and chromatographed (hexanes-EtOAc $=10: 1)$ to give $\mathbf{3}(18.9 \mathrm{mg}, 90 \%)$ as yellow solid. TLC (hexanes-EtOAc $=3: 1): R_{f}=0.5 .{ }^{1} \mathrm{H}$ NMR: $\delta 8.12(\mathrm{~d}, J=9.2 \mathrm{~Hz}, 2 \mathrm{H}), 7.51(\mathrm{~d}, J=9.2 \mathrm{~Hz}$, $2 \mathrm{H}), 7.32-7.16(\mathrm{~m}, 11 \mathrm{H}), 5.94(\mathrm{~m}, 1 \mathrm{H}), 5.49(\mathrm{~d}, J=17.2 \mathrm{~Hz}, 1 \mathrm{H}), 5.40(\mathrm{~d}, J=10.0 \mathrm{~Hz}, 1$ H), $4.96(\mathrm{~d}, J=11.6 \mathrm{~Hz}, 1 \mathrm{H}), 4.06(\mathrm{~m}, 1 \mathrm{H}), 3.74(\mathrm{~d}, J=11.6 \mathrm{~Hz}, 1 \mathrm{H}), 2.91(\mathrm{dd}, J=6.0$, $11.6 \mathrm{~Hz}, 1 \mathrm{H}), 2.63(\mathrm{dd}, J=10.0,12.0 \mathrm{~Hz}, 1 \mathrm{H}) .{ }^{13} \mathrm{C}\left\{{ }^{1} \mathrm{H}\right\} \mathrm{NMR}(125 \mathrm{MHz}): \delta 153.3,145.2$, 144.7, 144.2, 142.2, 140.0, 128.7, 128.6, 126.7, 126.6, 126.56, 126.3, 125.0, 118.5, 117.8, 
59.8, 55.5, 51.8, 46.0. IR (neat, $\mathrm{cm}^{-1}$ ): 2922, 1679, 1547, 1501, 1327, 1254, 752, 704.

HRMS calcd (found) for $\mathrm{C}_{25} \mathrm{H}_{24} \mathrm{~N}_{3} \mathrm{O}_{3}\left(\mathrm{MH}^{+}\right)$: 414.1818 (414.1827). Anal. calcd (found) for $\mathrm{C}_{25} \mathrm{H}_{23} \mathrm{~N}_{3} \mathrm{O}_{3}$ : H, 5.61 (5.55); C, 72.62 (72.62).

Remaining heterocycles were synthesized employing procedures similar to that used to synthesize 3. Reaction temperature, time, and solvent system are noted in Tables 2 and 3.

4.2.2. N,4,4-Triphenyl-2-vinylpyrrolidine-1-carboxamide (Table 2, entry 2)White solid, 98\%. TLC (hexanes-EtOAc $=3: 1): R_{f}=0.4 .{ }^{1} \mathrm{H}$ NMR: $\delta 7.37(\mathrm{~d}, J=9.6 \mathrm{~Hz}, 2$ H), 7.32-7.16 (m, $12 \mathrm{H}), 7.00(\mathrm{dt}, J=1.2,7.6 \mathrm{~Hz}, 1 \mathrm{H}), 6.70(\mathrm{~s}, 1 \mathrm{H}), 5.92(\mathrm{~m}, 1 \mathrm{H}), 5.43(\mathrm{~d}$, $J=13.2 \mathrm{~Hz}, 1 \mathrm{H}), 5.33(\mathrm{~d}, J=10.0 \mathrm{~Hz}, 1 \mathrm{H}), 4.99(\mathrm{~d}, J=11.6 \mathrm{~Hz}, 1 \mathrm{H}), 4.01(\mathrm{q}, J=8.8 \mathrm{~Hz}$, $1 \mathrm{H}), 3.72(\mathrm{~d}, J=12.0 \mathrm{~Hz}, 1 \mathrm{H}), 2.86(\mathrm{q}, J=8.4 \mathrm{~Hz}, 1 \mathrm{H}), 2.62(\mathrm{dd}, J=9.6,12.0 \mathrm{~Hz}, 1$ H). ${ }^{13} \mathrm{C}\left\{{ }^{1} \mathrm{H}\right\}$ NMR: $\delta 154.4,145.1,144.6,140.4,139.1,128.8,128.7,128.5,126.7,126.6$, 126.5, 126.4, 122.8, 119.0, 117.8, 59.6, 55.4, 51.8, 46.3. IR (neat, $\mathrm{cm}^{-1}$ ): 2925, 1653, 1533, $1443,1367,1245,753,697$. Anal. calcd (found) for $\mathrm{C}_{25} \mathrm{H}_{24} \mathrm{~N}_{2} \mathrm{O}: \mathrm{H}, 6.57$ (6.52); C, 81.49 (81.25).

4.2.3. N-(4-lodophenyl)-4,4-diphenyl-2-vinylpyrrolidine-1-carboxamide (Table 2, entry 3)—White solid, 88\%. TLC (hexanes-EtOAc $=3: 1$ ): $R_{f}=0.6 .{ }^{1} \mathrm{H}$ NMR: $\delta 7.55$ $(\mathrm{m}, 2 \mathrm{H}), 7.33-7.14(\mathrm{~m}, 12 \mathrm{H}), 5.92(\mathrm{~m}, 1 \mathrm{H}), 5.44(\mathrm{~d}, J=13.2 \mathrm{~Hz}, 1 \mathrm{H}), 5.34(\mathrm{~d}, J=10.0$ $\mathrm{Hz}, 1 \mathrm{H}), 4.97(\mathrm{dd}, J=1.6,11.6 \mathrm{~Hz}, 1 \mathrm{H}), 4.00(\mathrm{~m}, 1 \mathrm{H}), 3.71(\mathrm{~d}, J=11.6 \mathrm{~Hz}, 1 \mathrm{H}), 2.87$ $(\mathrm{dq}, J=2.0,6.4 \mathrm{~Hz}, 1 \mathrm{H}), 2.62(\mathrm{dd}, J=9.2,12.4 \mathrm{~Hz}, 1 \mathrm{H}) .{ }^{13} \mathrm{C}\left\{{ }^{1} \mathrm{H}\right\}$ NMR: $\delta 154.1,145.0$, 144.5, 140.4, 138.9, 137.7, 128.7, 128.6, 126.7, 126.6, 126.5, 120.9, 118.1, 85.4, 59.7, 55.4, 51.8, 46.3. IR (neat, $\mathrm{cm}^{-1}$ ): 2925, 1654, 1521, 1398, 1366, 1241, 699. Anal. calcd (found) for $\mathrm{C}_{25} \mathrm{H}_{24} \mathrm{~N}_{2} \mathrm{O}: \mathrm{H}, 4.69$ (4.67); C, 60.74 (60.96).

\subsubsection{1-(3,5-bis(Trifluoromethyl)phenyl)-3-(2,2-diphenylhexa-4,5-dienyl)urea} (Table 2, entry 4)—White solid, 92\%. TLC (hexanes-EtOAc $=3: 1$ ): $R_{f}=0.60 .{ }^{1} \mathrm{H}$ NMR: $\delta 7.84(\mathrm{~s}, 2 \mathrm{H}), 7.48(\mathrm{~s}, 1 \mathrm{H}), 7.33-7.17(\mathrm{~m}, 10 \mathrm{H}), 6.99(\mathrm{~s}, 1 \mathrm{H}), 5.94(\mathrm{~m}, 1 \mathrm{H}), 5.49(\mathrm{~d}, J=$ $17.2 \mathrm{~Hz}, 1 \mathrm{H}), 5.41(\mathrm{~d}, J=10.0 \mathrm{~Hz}, 1 \mathrm{H}), 4.97(\mathrm{~d}, J=11.6 \mathrm{~Hz}, 1 \mathrm{H}), 4.04(\mathrm{q}, J=8.0 \mathrm{~Hz}, 1$ H), $3.74(\mathrm{~d}, J=11.6 \mathrm{~Hz}, 1 \mathrm{H}), 2.90(\mathrm{q}, J=6.4 \mathrm{~Hz}, 1 \mathrm{H}), 2.64(\mathrm{dd}, J=9.6,12.0 \mathrm{~Hz}, 1$ H). ${ }^{13} \mathrm{C}\left\{{ }^{1} \mathrm{H}\right\}$ NMR: $\delta 153.7,144.8,144.3,140.6,140.1,132.6,132.3,131.9,131.6,128.8$, 128.7, 126.8, 126.7, 126.6, 126.4, 124.5, 121.8, 118.6, 116.0, 59.8, 55.5, 51.8, 46.2. IR (neat, $\mathrm{cm}^{-1}$ ): 1656, 1474, 1364, 1277, 1176, 1129, 670. Anal. calcd (found) for $\mathrm{C}_{27} \mathrm{H}_{22} \mathrm{~N}_{2} \mathrm{O}$ : H, 4.40 (4.38); C, 64.28 (64.53).

\subsubsection{N-(4-Methoxyphenyl)-4,4-diphenyl-2-vinylpyrrolidine-1-carboxamide} (Table 2, entry 5)—White solid, 90\%. TLC (hexanes-EtOAc $=1: 1$ ): $R_{f}=0.6 .{ }^{1} \mathrm{H}$ NMR: $\delta$ 7.31-7.17 (m, $12 \mathrm{H}), 6.81(\mathrm{~d}, J=8.8 \mathrm{~Hz}, 2 \mathrm{H}), 6.52(\mathrm{~s}, 1 \mathrm{H}), 5.91(\mathrm{~m}, 1 \mathrm{H}), 5.40(\mathrm{~d}, J=17.2$ $\mathrm{Hz}, 1 \mathrm{H}), 5.30(\mathrm{~d}, J=10.0 \mathrm{~Hz}, 1 \mathrm{H}), 4.97(\mathrm{~d}, J=11.6 \mathrm{~Hz}, 1 \mathrm{H}), 3.76(\mathrm{~s}, 1 \mathrm{H}), 3.70(\mathrm{~d}, J=$ $11.6 \mathrm{~Hz}, 1 \mathrm{H}), 2.84(\mathrm{dq}, J=1.2,6.0 \mathrm{~Hz}, 1 \mathrm{H}), 2.60(\mathrm{dd}, J=9.6,12.0 \mathrm{~Hz}, 1 \mathrm{H}) .{ }^{13} \mathrm{C}\left\{{ }^{1} \mathrm{H}\right\}$ NMR: $\delta$ 155.5, 154.8, 145.2, 144.7, 140.5, 132.2, 128.7, 128.5, 126.7, 126.6, 126.5, 126.4, 121.2, 117.6, 114.1, 59.6, 55.5, 51.9, 46.4. IR (neat, $\mathrm{cm}^{-1}$ ): 2930, 1650, 1531, 1512, 1367, 1235, 1034, 827, 701. Anal. calcd (found) for $\mathrm{C}_{26} \mathrm{H}_{26} \mathrm{~N}_{2} \mathrm{O}_{2}: \mathrm{H}, 6.58$ (6.77); C, 78.36 (78.26).

\subsubsection{N-(4-Methoxybenzyl)-4,4-diphenyl-2-vinylpyrrolidine-1-carboxamide} (Table 2, entry 6)—White solid, 89\%. TLC (hexanes-EtOAc $=1: 1): R_{f}=0.45 .{ }^{1} \mathrm{H}$ NMR: $\delta$ 7.29-7.13 (m, $12 \mathrm{H}), 6.81(\mathrm{~d}, J=8.4 \mathrm{~Hz}, 2 \mathrm{H}), 5.79(\mathrm{~m}, 1 \mathrm{H}), 5.19(\mathrm{~d}, J=17.2 \mathrm{~Hz}, 1 \mathrm{H})$, $5.12(\mathrm{~d}, J=10.0 \mathrm{~Hz}, 1 \mathrm{H}), 4.95(\mathrm{~d}, J=11.6 \mathrm{~Hz}, 1 \mathrm{H}), 4.82(\mathrm{t}, J=5.6 \mathrm{~Hz}, 1 \mathrm{H}), 4.35(\mathrm{~m}, 2$ H), $3.84(\mathrm{q}, J=8.0 \mathrm{~Hz}, 1 \mathrm{H}), 3.78(\mathrm{~s}, 1 \mathrm{H}), 3.62(\mathrm{~d}, J=11.6 \mathrm{~Hz}, 1 \mathrm{H}), 2.76(\mathrm{q}, J=6.4 \mathrm{~Hz}, 1$ H), $2.53(\mathrm{dd}, J=9.6,12.0 \mathrm{~Hz}, 1 \mathrm{H}) .{ }^{13} \mathrm{C}\left\{{ }^{1} \mathrm{H}\right\}$ NMR: $\delta 158.7,157.3,145.3,144.9,140.2$, $131.7,128.60,128.56,128.50,126.8,126.6,126.5,126.4,117.0,113.9,59.2,55.7,55.3$, 
52.1, 46.4, 44.0. IR (neat, $\mathrm{cm}^{-1}$ ): 2925, 1638, 1512, 1246, 1176, 1033, 701. Anal. calcd (found) for $\mathrm{C}_{27} \mathrm{H}_{28} \mathrm{~N}_{2} \mathrm{O}_{2}: \mathrm{H}, 6.84$ (6.74); $\mathrm{C}, 78.61$ (78.53).

4.2.7. N-Butyl-4,4-diphenyl-2-vinylpyrrolidine-1-carboxamide (Table 2, entry 7) -White solid, 90\%. TLC (hexanes-EtOAc $=3: 1$ ): $R_{f}=0.4 .{ }^{1} \mathrm{H}$ NMR: $\delta$ 7.29-7.14 (m, 10 $\mathrm{H}), 5.80(\mathrm{~m}, 1 \mathrm{H}), 5.25(\mathrm{~d}, J=17.2 \mathrm{~Hz}, 1 \mathrm{H}), 5.17(\mathrm{~d}, J=10.4 \mathrm{~Hz}, 1 \mathrm{H}), 4.91(\mathrm{dd}, J=1.2$, $11.6 \mathrm{~Hz}, 1 \mathrm{H}), 4.51(\mathrm{t}, J=5.6 \mathrm{~Hz}, 1 \mathrm{H}), 3.80(\mathrm{q}, J=8.0 \mathrm{~Hz}, 1 \mathrm{H}), 3.60(\mathrm{~d}, J=11.2 \mathrm{~Hz}, 1 \mathrm{H})$, $3.21(\mathrm{~m}, 1 \mathrm{H}), 2.74(\mathrm{dq}, J=2.0,6.0 \mathrm{~Hz}, 1 \mathrm{H}), 2.53(\mathrm{dd}, J=9.6,12.0 \mathrm{~Hz}, 1 \mathrm{H}), 1.41(\mathrm{~m}, 2$ H), 1.27 (m, $2 \mathrm{H}), 0.89$ (t, $J=7.6 \mathrm{~Hz}, 3 \mathrm{H}) .{ }^{13} \mathrm{C}\left\{{ }^{1} \mathrm{H}\right\}$ NMR: $\delta 157.5,145.4,145.1,140.5$, 128.6, 128.5, 126.8, 126.6, 126.5, 126.3, 116.7, 59.3, 55.6, 52.0, 46.5, 40.2, 32.2, 20.0, 13.8 . IR (neat, $\mathrm{cm}^{-1}$ ): 2928, 1634, 1529, 1358, 1205, 756, 700. Anal. calcd (found) for $\mathrm{C}_{23} \mathrm{H}_{28} \mathrm{~N}_{2} \mathrm{O}: \mathrm{H}, 8.10$ (8.14); C, 79.27 (79.12).

4.2.8. 2-(2-Methylprop-1-enyl)-N-(4-nitrophenyl)-4,4-diphenylpyrrolidine-1carboxamide (Table 2, entry 8)-Yellow solid, 91\%. TLC (hexanes-EtOAc = 3:1): $R_{f}=$ 0.4. ${ }^{1} \mathrm{H}$ NMR: $\delta 8.13$ (d, $\left.J=9.2 \mathrm{~Hz}, 2 \mathrm{H}\right), 7.41(\mathrm{~d}, J=8.0 \mathrm{~Hz}, 2 \mathrm{H}), 7.31-7.17(\mathrm{~m}, 10 \mathrm{H})$, $7.14(\mathrm{~s}, 1 \mathrm{H}), 5.31(\mathrm{~d}, J=9.2 \mathrm{~Hz}, 1 \mathrm{H}), 4.97(\mathrm{~d}, J=11.6 \mathrm{~Hz}, 1 \mathrm{H}), 4.24(\mathrm{~m}, 1 \mathrm{H}), 3.66(\mathrm{~d}, J=$ $12.0 \mathrm{~Hz}, 1 \mathrm{H}), 2.81(\mathrm{q}, J=6.0 \mathrm{~Hz}, 1 \mathrm{H}), 2.54(\mathrm{dd}, J=9.6,12.0 \mathrm{~Hz}, 1 \mathrm{H}) .{ }^{13} \mathrm{C}\left\{{ }^{1} \mathrm{H}\right\}$ NMR: $\delta$ 153.3, 145.4, 145.1, 144.5, 142.1, 137.7, 128.8, 128.6, 126.7, 126.64, 126.60, 126.4, 126.1, 125.2, 117.6, 55.1, 54.6, 51.7, 45.8, 26.0, 18.1. IR (neat, $\mathrm{cm}^{-1}$ ): 2922, 1681, 1540, 1504, 1328, 1254, 1113, 699. Anal. calcd (found) for $\mathrm{C}_{27} \mathrm{H}_{27} \mathrm{~N}_{3} \mathrm{O}_{3}: \mathrm{H}, 6.16$ (6.20); C, 73.45 (73.60).

4.2.9. $\mathbf{N}$-(4-Nitrophenyl)-5,5-diphenyl-2-vinylpiperidine-1-carboxamide (Table 2 , entry 9)-Yellow solid, 89\%. TLC (hexanes-EtOAc $=3: 1$ ): $R_{f}=0.5 .{ }^{1} \mathrm{H}$ NMR: $\delta 8.15$ (d, $J$ $=9.2 \mathrm{~Hz}, 2 \mathrm{H}), 7.47(\mathrm{~d}, J=9.2 \mathrm{~Hz}, 2 \mathrm{H}), 7.33-7.16(\mathrm{~m}, 10 \mathrm{H}), 6.69(\mathrm{~s}, 1 \mathrm{H}), 5.89(\mathrm{~m}, 1 \mathrm{H})$, $5.40(\mathrm{dd}, J=2.0,10.8 \mathrm{~Hz}, 1 \mathrm{H}), 5.23(\mathrm{dd}, J=2.0,17.6 \mathrm{~Hz}, 1 \mathrm{H}), 5.10(\mathrm{dd}, J=2.0,14.0 \mathrm{~Hz}$, $1 \mathrm{H}), 4.48(\mathrm{~m}, 1 \mathrm{H}), 3.10(\mathrm{~d}, J=14.0 \mathrm{~Hz}, 1 \mathrm{H}), 2.49(\mathrm{dq}, J=2.8,13.2 \mathrm{~Hz}, 1 \mathrm{H}), 2.36(\mathrm{dt}, J=$ 4.0, $12.8 \mathrm{~Hz}, 1 \mathrm{H}), 1.85-1.77(\mathrm{~m}, 2 \mathrm{H}) .{ }^{13} \mathrm{C}\left\{{ }^{1} \mathrm{H}\right\}$ NMR (125 MHz): $\delta$ 154.3, 147.1, 145.3 , 144.2, 135.3, 128.6, 128.5, 127.7, 126.6, 126.2, 125.0, 118.6, 117.5, 53.5, 48.0, 46.3, 29.9, 25.9. IR (neat, $\mathrm{cm}^{-1}$ ): 1724, 1654, 1501, 1330, 1251, 1112, 752, 701. HRMS calcd (found) for $\mathrm{C}_{26} \mathrm{H}_{26} \mathrm{~N}_{3} \mathrm{O}_{3}\left(\mathrm{MH}^{+}\right)$: 428.1974 (428.1987). Anal. calcd (found) for $\mathrm{C}_{26} \mathrm{H}_{25} \mathrm{~N}_{3} \mathrm{O}_{3}: \mathrm{H}$, 5.89 (5.84); C, 73.05 (72.79).

4.2.10. N-(4-Nitrophenyl)-3-vinyl-2-aza-spiro[4.5]decane-2-carboxamide (Table 2, entry 10)-Yellow solid, 76\%. TLC (hexanes-EtOAc $=3: 1$ ): $R_{f}=0.3 .{ }^{1} \mathrm{H}$ NMR: $\delta 8.12$ (m, $2 \mathrm{H}), 7.49(\mathrm{~m}, 2 \mathrm{H}), 7.09(\mathrm{~s}, 1 \mathrm{H}), 5.94(\mathrm{~m}, 1 \mathrm{H}), 5.47(\mathrm{~d}, J=17.2 \mathrm{~Hz}, 1 \mathrm{H}), 5.36(\mathrm{~d}, J=$ $10.0 \mathrm{~Hz}, 1 \mathrm{H}), 4.26(\mathrm{q}, J=8.0 \mathrm{~Hz}, 1 \mathrm{H}), 3.80(\mathrm{~d}, J=11.2 \mathrm{~Hz}, 1 \mathrm{H}), 3.14(\mathrm{~d}, J=11.2 \mathrm{~Hz}, 1$ H), $2.15(\mathrm{dq}, J=1.2,7.2 \mathrm{~Hz}, 1 \mathrm{H}), 1.62(\mathrm{dd}, J=8.8,12.8 \mathrm{~Hz}, 1 \mathrm{H}), 1.50-1.32(\mathrm{~m}, 10$ H). ${ }^{13} \mathrm{C}\left\{{ }^{1} \mathrm{H}\right\}$ NMR: $\delta 153.6,145.4,142.2,140.9,125.1,117.7,117.2,59.8,56.9,45.6,40.5$, 36.3, 34.6, 26.0, 23.7, 22.9. IR (neat, $\mathrm{cm}^{-1}$ ): 2924, 1664, 1543, 1499, 1328, 1250, 1113, 854, 752, 609. Anal. calcd (found) for $\mathrm{C}_{18} \mathrm{H}_{23} \mathrm{~N}_{3} \mathrm{O}_{3}: \mathrm{H}, 7.04$ (7.05); C, 65.63 (65.42).

4.2.11. N-(4-Nitrophenyl)-2-vinylpyrrolidine-1-carboxamide (Table 2, entry 11) -Yellow solid, 82\%. TLC (hexanes-EtOAc $=1: 1): R_{f}=0.5 .{ }^{1} \mathrm{H}$ NMR: $\delta 8.12(\mathrm{~d}, J=9.2$ $\mathrm{Hz}, 2 \mathrm{H}), 7.49(\mathrm{~d}, J=9.2 \mathrm{~Hz}, 2 \mathrm{H}), 6.88(\mathrm{~s}, 1 \mathrm{H}), 5.91(\mathrm{~m}, 1 \mathrm{H}), 5.35(\mathrm{~d}, J=17.2 \mathrm{~Hz}, 1 \mathrm{H})$, $5.12(\mathrm{~d}, J=10.0 \mathrm{~Hz}, 1 \mathrm{H}), 4.28(\mathrm{~m}, 1 \mathrm{H}), 3.64(\mathrm{dt}, J=7.2,10.4 \mathrm{~Hz}, 1 \mathrm{H}), 3.54(\mathrm{~m}, 1 \mathrm{H})$, 2.25-2.17 (m, $1 \mathrm{H}), 1.99-1.81(\mathrm{~m}, 3 \mathrm{H}) .{ }^{13} \mathrm{C}\left\{{ }^{1} \mathrm{H}\right\}$ NMR: $\delta 153.2,145.4,142.2,139.2,125.1$, 117.8, 116.7, 60.1, 47.0, 33.2, 22.9. IR (neat, $\mathrm{cm}^{-1}$ ): 1658, 1501, 1328, 1251, 1112, 852, 751. Anal. calcd (found) for $\mathrm{C}_{13} \mathrm{H}_{15} \mathrm{~N}_{3} \mathrm{O}_{3}: \mathrm{H}, 5.79$ (5.85); C, 59.76 (59.39). 
4.2.12. 2-(2-Ethyloct-1-enyl)-N-(4-nitrophenyl)-4,4-diphenylpyrrolidine-1carboxamide (5)-Yellow solid, 93\% as a 1.3:1 mixture of diastereomers. TLC (hexanesEtOAc $=3: 1): R_{f}=0.65 .{ }^{1} \mathrm{H}$ NMR: $\delta 8.12(\mathrm{~m}, 2 \mathrm{H}), 7.42(\mathrm{~m}, 2 \mathrm{H}), 7.31-7.14(\mathrm{~m}, 11 \mathrm{H})$, [5.27 (d, $J=9.2 \mathrm{~Hz}), 5.23(\mathrm{~d}, J=9.2 \mathrm{~Hz}), 1.3: 1,1 \mathrm{H}], 4.95(\mathrm{~d}, J=11.6 \mathrm{~Hz}, 1 \mathrm{H}), 4.28(\mathrm{~m}, 1$ $\mathrm{H}),[3.73(\mathrm{~d}, J=12.0 \mathrm{~Hz}), 3.72(\mathrm{~d}, J=12.0 \mathrm{~Hz}), 1.3: 1,1 \mathrm{H}], 2.82(\mathrm{~m}, 1 \mathrm{H}), 2.56(\mathrm{~m}, 1 \mathrm{H})$, 2.22-1.98 (m, $4 \mathrm{H}), 1.44-1.19(\mathrm{~m}, 8 \mathrm{H}),[1.03(\mathrm{t}, J=7.6 \mathrm{~Hz}), 1.01(\mathrm{t}, J=7.6 \mathrm{~Hz}), 1.3: 1,3 \mathrm{H}]$, $0.84(\mathrm{t}, J=7.2 \mathrm{~Hz}, 3 \mathrm{H}) .{ }^{13} \mathrm{C}\left\{{ }^{1} \mathrm{H}\right\}$ NMR: $\delta 153.4,147.7,145.4,145.1,145.0,144.4,142.2$, 129.1, 128.8, 128.6, 126.7, 126.6, 126.4, 126.3, 125.2, 124.8, 117.6, 117.5, 55.3, 55.2, 54.2, 51.7, 46.3, 36.4, 31.9, 31.8, 31.6, 31.4, 30.6, 29.7, 29.2, 29.1, 28.8, 28.1, 22.5, 22.4, 14.0, 13.7, 12.7. IR (neat, $\mathrm{cm}^{-1}$ ): 2928, 1680, 1541, 1503, 1327, 1253, 1112, 750, 699. Anal. calcd (found) for $\mathrm{C}_{33} \mathrm{H}_{39} \mathrm{~N}_{3} \mathrm{O}_{3}: \mathrm{H}, 7.48$ (7.26); C, 75.40 (75.27).

\subsection{Control Experiments}

4.3.1. Gold alone-A solution of $\mathbf{1}(21.0 \mathrm{mg}, 0.05 \mathrm{mmol})$, [(S)-2] $\mathrm{Au}_{2} \mathrm{Cl}_{2}(4.0 \mathrm{mg}, 5 \mathrm{~mol}$ $\%)$, and $n$-hexadecane ( $11 \mathrm{mg}$, internal standard) in ether $(0.5 \mathrm{~mL})$ was stirred at $23{ }^{\circ} \mathrm{C}$ for $48 \mathrm{~h}$. GC analysis of the crude reaction mixture revealed neither detectable consumption of $\mathbf{1}$ nor formation of $\mathbf{3}$.

4.3.2. Silver alone-A solution of $\mathbf{1}(21.0 \mathrm{mg}, 0.05 \mathrm{mmol}), \mathrm{AgBF}_{4}(1.0 \mathrm{mg}, 10 \mathrm{~mol} \%)$, and $n$-hexadecane $\left(11 \mathrm{mg}\right.$, internal standard) in ether $(0.5 \mathrm{~mL})$ was stirred at $23{ }^{\circ} \mathrm{C}$ for $48 \mathrm{~h}$. $\mathrm{GC}$ analysis of the crude reaction mixture revealed neither detectable consumption of $\mathbf{1}$ nor formation of $\mathbf{3}$.

4.3.3. Silver and ligand-A solution of $\mathbf{1}(21.0 \mathrm{mg}, 0.05 \mathrm{mmol}), \mathrm{AgBF}_{4}(1.0 \mathrm{mg}, 10 \mathrm{~mol}$ $\%),(S)-2(3.0 \mathrm{mg}, 5 \mathrm{~mol} \%)$, and $n$-hexadecane (11 mg, internal standard) in ether $(0.5 \mathrm{~mL})$ was stirred at $23{ }^{\circ} \mathrm{C}$ for $48 \mathrm{~h}$. GC analysis of the crude reaction mixture revealed neither detectable consumption of $\mathbf{1}$ nor formation of $\mathbf{3}$.

\section{Supplementary Material}

Refer to Web version on PubMed Central for supplementary material.

\section{Acknowledgments}

The authors thank the NIH (GM-080422) for support of this research.

\section{References}

1. (a) Müller TE, Hultzsch KC, Yus M, Foubelo F, Tada M. Chem. Rev. 2008; 108:3795-3892. [PubMed: 18729420] (b) Widenhoefer RA, Han X. Eur. J. Org. Chem. 2006:4555-4563.(c) Hong S, Marks TJ. Acc. Chem. Res. 2004; 37:673-686. [PubMed: 15379583]

2. (a) Walsh PJ, Baranger AM, Bergman RG. J. Am. Chem. Soc. 1992; 114:1708-1719.(b) Besson L, Gore J, Cazes B. Tetrahedron Lett. 1995; 36:3857-3860.(c) Al-Masum M, Meguro M, Yamamoto Y. Tetrahedron Lett. 1997; 38:6071-6074.(d) Johnson JS, Bergman RG. J. Am. Chem. Soc. 2001; 123:2923-2924. [PubMed: 11456996] (e) Nishina N, Yamamoto Y. Angew. Chem. Int. Ed. 2006; 45:3314-3317.(f) Ayinla RO, Schafer LL. Inorg. Chim. Acta. 2006; 359:3097-3102.(g) Nishina N, Yamamoto Y. Synlett. 2007:1767-1770.(h) Lavallo V, Frey GD, Donnadieu B, Soleilhavoup M, Bertrand G. Angew. Chem. Int. Ed. 2008; 47:5224-5228.(i) Kinder RE, Zhang Z, Widenhoefer RA. Org. Lett. 2008; 10:3157-3159. [PubMed: 18570376] (j) Zeng X, Soleilhavoup M, Bertrand G. Org. Lett. 2009; 11:3166-3169. [PubMed: 19719176] (k) Duncan AN, Widenhoefer RA. SYNLETT. 2010:419-422. [PubMed: 21197142] (1) Toups KL, Widenhoefer RA. Chem. Commun. 2010; 46:1712-1714.

3. (a) Claesson A, Sahlberg C, Luthman K. Acta Chem. Scand. 1979:309-310.(b) Hubry NJS, Kinsman RG, Lathbury D, Vernon PG, Gallagher T. J. Chem. Soc., Perkin Trans. 1991; 1:145-155. 
(c) Gallagher T, Jones SW, Mahon MF, Molloy KC. J. Chem. Soc., Perkin Trans. 1991; 1:21932198.(d) Arredondo VM, McDonald FE, Marks TJ. J. Am. Chem. Soc. 1998; 120:4871-4872.(e) Meguro M, Yamamoto Y. Tetrahedron Lett. 1998; 39:5421-5424.(f) Ha JD, Cha JK. J. Am. Chem. Soc. 1999; 121:10012-10020.(g) Ackermann L, Bergman RG. Org. Lett. 2002; 4:1475-1478a. [PubMed: 11975607] (h) Hong S, Kawaoka AM, Marks TJ. J. Am. Chem. Soc. 2003; 125:1587815892. [PubMed: 14677980] (i) Ackermann L, Bergman RG, Loy RN. J. Am. Chem. Soc. 2003; 125:11956-11963. [PubMed: 14505417] (j) Morita N, Krause N. Org. Lett. 2004; 6:4121-4123. [PubMed: 15496114] (k) Lee PH, Kim H, Lee K, Kim M, Noh K, Kim H, Seomoon D. Angew. Chem. Int. Ed. 2005; 44:1840-1843.(1) Morita N, Krause N. Eur. J. Org. Chem. 2006:4634-4641. (m) Zhang Z, Liu C, Kinder RE, Han X, Qian H, Widenhoefer RA. J. Am. Chem. Soc. 2006; 128:9066-9073. [PubMed: 16834380] (n) Volz F, Krause N. Org. Biomol. Chem. 2007; 5:15191521. [PubMed: 17571178] (o) Bates RW, Dewey MR. Org. Lett. 2009; 11:3706-3708. [PubMed: 19719203] (p) Manzo AM, Perboni AD, Broggini G, Rigamonti MM. Tetrahedron Lett. 2009; 50:4696-4699.(k) Nishina N, Yamamoto Y. Tetrahedron. 2009; 65:1799-1808.

4. (a) For examples of titanium-catalyzed enantioselective allene hydroamination see: Hoover JM, Petersen JR, Pikul JH, Johnson AR. Organometallics. 2004; 23:4614-4620. (b) Hickman AJ, Hughs LD, Jones CM, Li HH, Redford JE, Sobelman DJ, Kouzelos JA, Johnson AR. Tetrahedron Assym. 2009; 20:1279-1285.

5. (a) For reviews of enantioselective hydroamination see: (a) Zi G. Dalton Trans. 2009:9101. [PubMed: 20449181] (b) Aillaud I, Collin J, Hannedouche J, Schulz E. Dalton Trans. 2007:51055118. [PubMed: 17985016] (c) Hultzsch KC. Org. Biomol. Chem. 2005; 3:1819-1824. [PubMed: 15889160] (d) Hultzsch KC. Adv. Synth. Catal. 2005; 347:367-391. (e) Roesky PW, Muller TE. Angew. Chem. Int. Ed. 2003; 42:2708-2710.

6. Zhang Z, Bender CF, Widenhoefer RA. Org. Lett. 2007; 9:2887-2889. [PubMed: 17595096]

7. Zhang Z, Bender CF, Widenhoefer RA. J. Am. Chem. Soc. 2007; 129:14148-14149. [PubMed: 17967025]

8. (a) LaLonde RL, Sherry BD, Kang EJ, Toste FD. J. Am. Chem. Soc. 2007; 129:2452-2453. [PubMed: 17290999] (b) Hamilton GL, Kang EJ, Mba FD. Toste Science. 2007; 317:496-499.

9. LaLonde RL, Wang ZJ, Mba M, Lackner AD, Toste Angew FD. Chem. Int. Ed. 2010; 49:598-601.

10. (a) For recent reviews of enantioselective gold(I) catalysis see: Krause N, Bongers N. Angew. Chem. Int. Ed. 2008; 47:2178-2181. (b) Widenhoefer RA. Chem. Eur. J. 2008; 14:5382-5391. (c) Sengupta S, Shi X. Chem. Cat. Chem. 2010; 2:609-619.

11. Bender CF, Widenhoefer RA. Org. Lett. 2006; 8:5303-5305. [PubMed: 17078703]

12. Zhang Z, Lee SD, Widenhoefer RA. J. Am. Chem. Soc. 2009; 131:5372-5373. [PubMed: 19326908]

13. Li H, Widenhoefer RA. Org. Lett. 2009; 11:2671-2674. [PubMed: 19514795]

14. Jung ME, Piizzi G. Chem. Rev. 2005; 105:1735-1766. [PubMed: 15884788]

15. Zhang Z, Widenhoefer RA. Angew. Chem. Int. Ed. 2007; 46:283-285.

16. Owing to their overlapping ${ }^{1} \mathrm{H}$ NMR spectra, we were unable to assign the relative configurations of the major and minor diastereomers of $\mathbf{5}$. However, hydroamination of the corresponding $N-\gamma-$ allenyl carbamate catalyzed by $[(S)-2] \mathrm{Au}_{2} \mathrm{Cl}_{2} / \mathrm{AgClO}_{4}$ forms predominantly (4.3:1) the (Z)-2vinyl pyrrolidine, which suggests that the major diastereomer of 5 is also $Z$ [12].

17. Zhang Z, Widenhoefer RA. Angew. Chem. Int. Ed. 2007; 46:283-285. 
Table 1

Effect of solvent on the conversion of $\mathbf{1}$ to 3 catalyzed by a mixture of $[(S)-2] \mathrm{Au}_{2} \mathrm{Cl}_{2}$ and $\mathrm{AgBF}_{4}$ at room temperature.

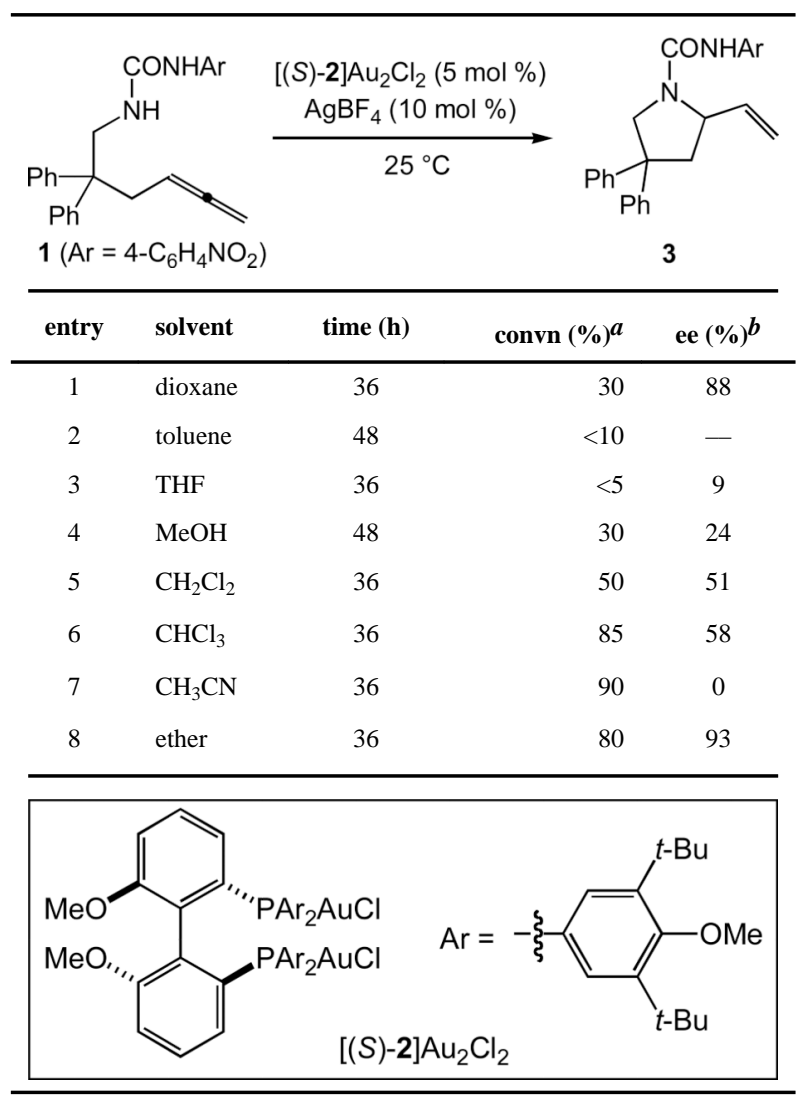

${ }^{a}$ Determined by GC analysis.

${ }^{b}$ Determined by HPLC analysis on a chiral stationary phase. 


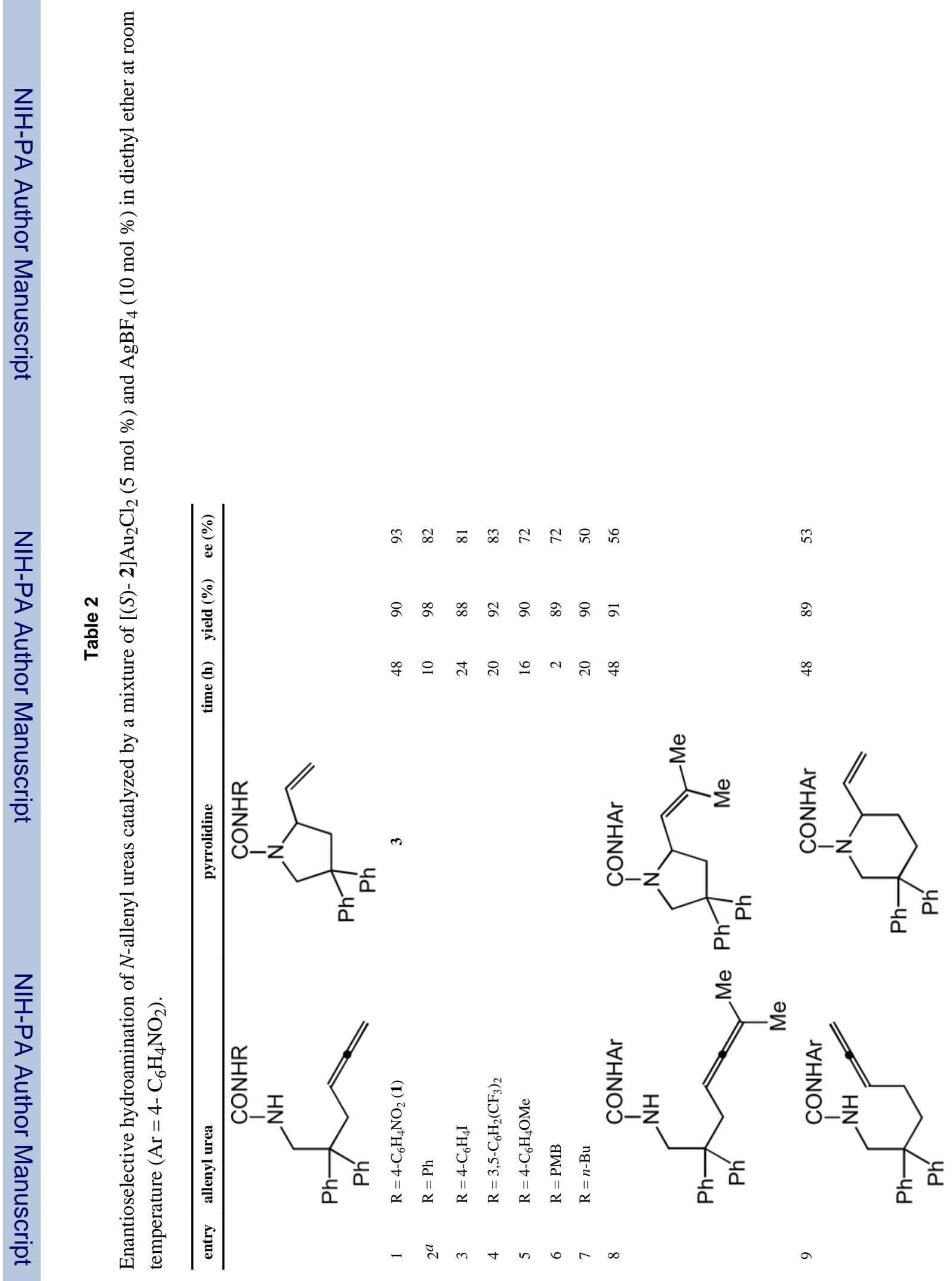


Li et al.

Page 10

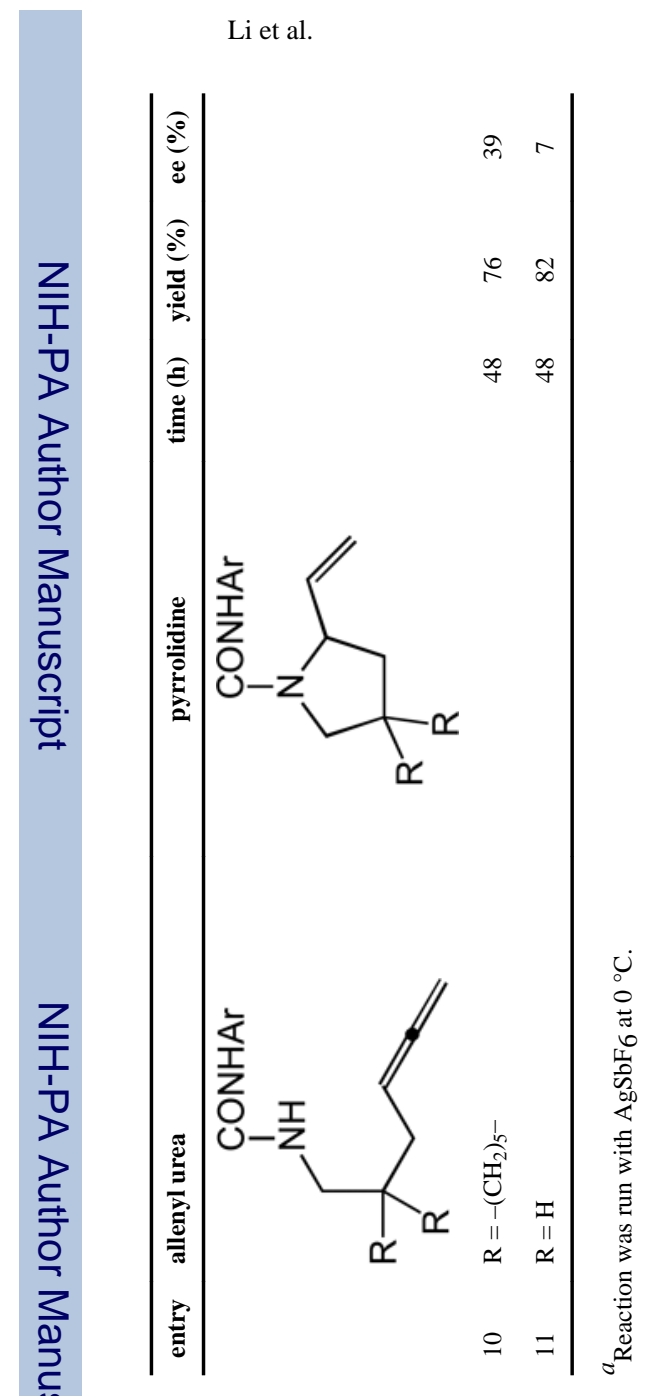

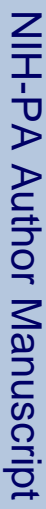

J Organomet Chem. Author manuscript; available in PMC 2011 September 7. 
